\title{
OPTIMIZATION OF THE PARAMETERS OF FEEDWATER CONTROL SYSTEM FOR OPR1000 NUCLEAR POWER PLANTS
}

\author{
UNG SOO KIM*, IN HO SONG, JONG JOO SOHN and EUN KEE KIM \\ Safety Analysis Department, KEPCO Engineering and Construction Company \\ 150, Deokjin-Dong, Yuseong-Gu, Daejeon, 305-353, Korea \\ ${ }^{*}$ Corresponding author. E-mail : uskim@kepco-enc.com
}

Received November 10, 2009

Accepted for Publication May 06, 2010

In this study, the parameters of the feedwater control system (FWCS) of the OPR1000 type nuclear power plant (NPP) are optimized by response surface methodology (RSM) in order to acquire better level control performance from the FWCS. The objective of the optimization is to minimize the steam generator (SG) water level deviation from the reference level during transients. The objective functions for this optimization are relationships between the SG level deviation and the parameters of the FWCS. However, in this case of FWCS parameter optimization, the objective functions are not available in the form of analytic equations and the responses (the SG level at plant transients) to inputs (FWCS parameters) can be evaluated by computer simulations only. Classical optimization methods cannot be used because the objective function value cannot be calculated directely. Therefore, the simulation optimization methodology is used and the RSM is adopted as the simulation optimization algorithm. Objective functions are evaluated with several typical transients in NPPs using a system simulation computer code that has been utilized for the system performance analysis of actual NPPs. The results show that the optimized parameters have better SG level control performance. The degree of the SG level deviation from the reference level during transients is minimized and consequently the control performance of the FWCS is remarkably improved.

KEYWORDS : Feedwater Control System, Parameter Optimization, Simulation Optimization, Response Surface Methodology, Steam Generator Level Control

\section{INTRODUCTION}

There are many control systems for the nuclear steam supply system (NSSS) in nuclear power plants (NPPs). These control systems make the NPP operate efficiently under transient conditions as well as under steady state conditions. The control performance of the NSSS control systems depends on the system's own control algorithms and on the relevant parameters such as gains, time constants, and so on. Among the NSSS control systems, the feedwater control system (FWCS) automatically regulates the steam generator (SG) downcomer water level to the desired setpoint by controlling the feedwater flow during NPP operations under not only steady state but also transient conditions. The main controller of the FWCS is a proportional-integral (PI) controller that includes a gain and a reset time constant as main parameters. The SG level error between the measured and the reference level (level setpoint) is compensated by feedwater and steam flow signal then sent to the PI controller and the resultant control demand signal is produced. This control demand is transferred to the feedwater control valves and feedwater pumps. The control performance of the FWCS dominantly depends on the gain and reset time constant of the main PI controller as mentioned above. So far, these parameters have been determined based on sensitivity studies using system simulation computer code or engineering judgments. This means that these parameters have not been optimized and that there is a room for improvement of the FWCS if these parameters come to have optimal values. In the OPR1000 type NPPs, the gain and reset time constant of the FWCS are not fixed at one value but are formed into piecewise linear functions that consist of several linear functions. In order to find the best values for these parameters including piecewise linear function points, all of these parameters are varied simultaneously to observe responses during the process to search for the best values. However, the sensitivity study has limits on its searching for the best values because the sensitivity study has difficulty in varying several parameters simultaneously to identify responses.

In order to solve this problem and find the best parameter values for the FWCS, the optimization that can handle several parameters simultaneously is suggested and performed. The objective of this optimization is to minimize the SG water level deviation from the reference level 
during transients. Hence the objective functions for this optimization are relationships between the SG level deviation and the parameters of the FWCS. However, in this case, the objective functions are not available in the form of analytic equations and the responses to inputs can be evaluated by computer simulations only. Therefore, a simulation optimization methodology is used.

\section{SIMULATION OPTIMIZATION}

The process of finding the best input variable value among all possibilities without explicitly evaluating each possibility is simulation optimization [1]. Simply stated, a simulation optimization problem is an optimization problem in which the objective function (objective functions, in case of a multi-criteria problem) and/or some constraints are responses that can only be evaluated by computer simulation. There are advantages in using simulation in optimization. In particular: Firstly, the complexity of the system being modeled does not significantly affect the performance of the optimization process. Secondly, for stochastic systems, the variance of the response is controllable by various output analysis techniques. Finally, where structural optimization of systems is considered, simulation provides an advantage that is often not possible in classical optimization procedures. Here, by employing appropriate techniques, the objective function or constraint can be changed from one iteration to another to reflect alternative design for the system [2].

The formulation of simulation optimization problems is often made for maximization or minimization of the objective function value of the system. A general simulation model comprises $n$ input variables $\left(x_{1}, x_{2}, \ldots, x_{n}\right)$ and $m$ output variables $\left(f_{1}(\mathrm{x}), f_{2}(\mathrm{x}), \ldots, f_{m}(\mathrm{x})\right)$ or $\left(y_{1}, y_{2}, \ldots, y_{m}\right)$ as shown in Fig. 1. Simulation optimization entails finding optimal settings of the input variables, i.e. values of $x_{1}, x_{2}, \ldots, x_{n}$, that optimize the output variables. A simulation optimization model is displayed in Fig. 2. The output of a simulation model is used by an optimization strategy to provide feedback on the progress of the search for the optimal solution. This in turn guides further input to the simulation model. In this work, the input and output correspond to FWCS parameters and the SG level at plant transients, respectively.

Simulation optimization methodologies are classified into six major categories. These are the gradient-based search method, the stochastic optimization, the response surface methodology (RSM), the heuristic method, the A-teams (asynchronous team), and the statistical methods [1]. Among these methods, the RSM is used for the optimization of the FWCS parameters because the RSM utilizes useful regression models that can be easily manipulated by a designer. It also smoothes out high frequency noises of an objective function and is thus expected to find a solution near the global optimum. In addition, it allows various objectives and constraints without additional numerical computations [3].

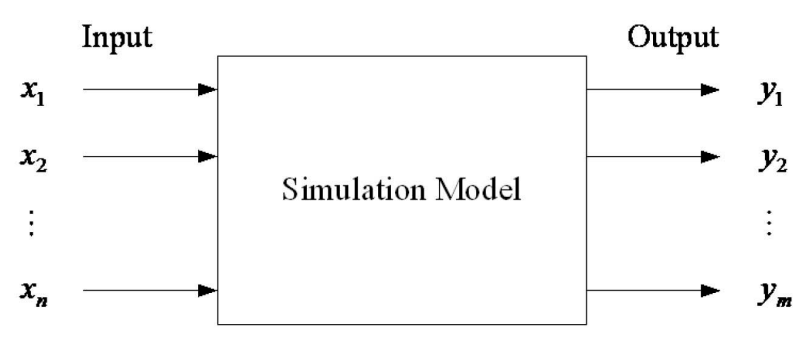

Fig. 1. General Simulation Model

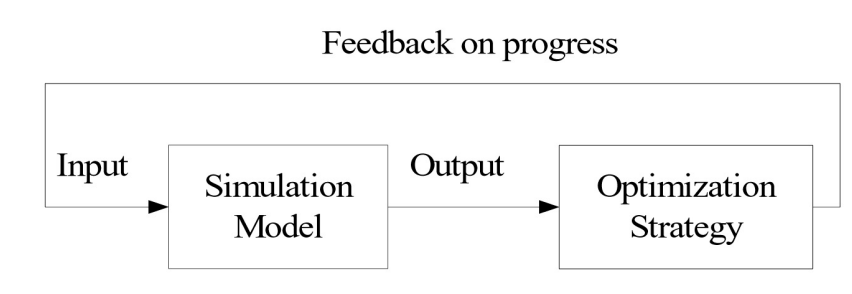

Fig. 2. Simulation Optimization Model

The RSM is a procedure for continual fitting of regression models to the output variable of a simulation model by evaluating it at several input variable values and optimizing the resulting regression function. It is a collection of statistical and mathematical techniques [3]. Most applications of RSM are sequential in nature. Phase zero is the screening experiment. This phase reduces a long list of variables to a relatively short one so that subsequent experiments will be more efficient and require fewer runs or tests. Once the important independent variables are identified, phase one of the RSM begins. In this phase, the experimenter's objective is to determine if the current levels of the independent variables result in a value of the response that is near the optimum or not. If the levels of the independent variables are not consistent with optimum performance, then the experimenter must determine a set of adjustments to the process variables that will move the process toward the optimum. This phase of the RSM makes considerable use of the first order model and an optimization technique called the method of steepest ascent/descent. Phase two of the RSM begins when the process is near the optimum. Because the true response surface usually exhibits curvature near the optimum, a second (or higher) order model will be used, and the obtained model is analyzed to determine the optimum conditions for the process [4]. The first order model used in phase one is given by Eq. (1) and Eq. (2) describes the second order model used in phase two. These are scalar equations.

$$
Y=b_{0}+\sum_{i=1}^{k} b_{i} x_{i}
$$




$$
Y=b_{0}+\sum_{i=1}^{k} b_{i} x_{i}+\sum_{i=1}^{k} \sum_{j=1}^{k} b_{i, j} x_{i} x_{j}
$$

where

$Y:$ response

$x_{i}$ : design variables

$b_{i}, b_{i, j}$ : regression coefficients

This sequential process is usually performed within a region of the independent variable space called the operability region [4].

\section{OPTIMIZATION OF THE PARAMETERS OF THE FWCS USING RSM}

The optimization of the parameters of the FWCS is performed to provide the FWCS with optimal performance for the SG level control. The objective of the optimization is to minimize level deviation from the target level setpoint during transient in NPP operation. The FWCS of the Ulchin NPP units (UCN) 5 and 6 is chosen for this study. There are two SGs in the UCN 5 and 6, and the target setpoint for $\mathrm{SG}$ level is $44 \%$ of narrow span. The parameters to be optimized in the FWCS are the master PI controller gain $K$ and the reset time constant $\tau$ as shown in Fig. 3. In the FWCS for the OPR1000 type NPP, like the UCN 5 and 6, the gain $K$ and the reset time constant $\tau$ are given as piecewise linear functions of the reactor power as shown in Fig. 4 and Fig. 5. As mentioned before, there is no analytic objective function for this optimization. The response for input can be evaluated by computer simulation only, and the optimization is performed using the RSM.

\subsection{Optimization of the Parameters of the FWCS}

The optimization of the gain and reset time of the FWCS is done for two regions. As mentioned before, the gain and reset time are the piecewise linear functions of the reactor power. Also, the operation characteristics of the FWCS at high reactor power $(\geq 20 \%)$ and low reactor power $(<20 \%)$ are different from each other as follows: For SG level control, the feedwater flow is controlled and two valves are used. These are the economizer valve (it is a large size valve) and the downcomer valve (it is a small size valve). At high reactor power, the feedwater flow is controlled by the economizer valve and the downcomer valve opening is fixed at the biased value. At low reactor power, the feedwater flow is controlled by the downcomer valve and the economizer valve is closed and not used. Therefore, the optimization of the gain and the reset time for high reactor power and low reactor power are performed separately. Through the optimization, the values for the piecewise points of the gain and the reset time are determined. The number of piecewise linear gain points is chosen as five (5) and that of the reset time point is chosen as one (1). Therefore, there are total six (6) (5 gain points and 1

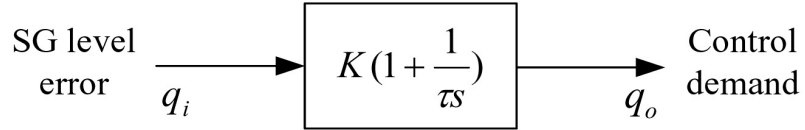

Fig. 3. Typical Model for FWCS Master PI Controller

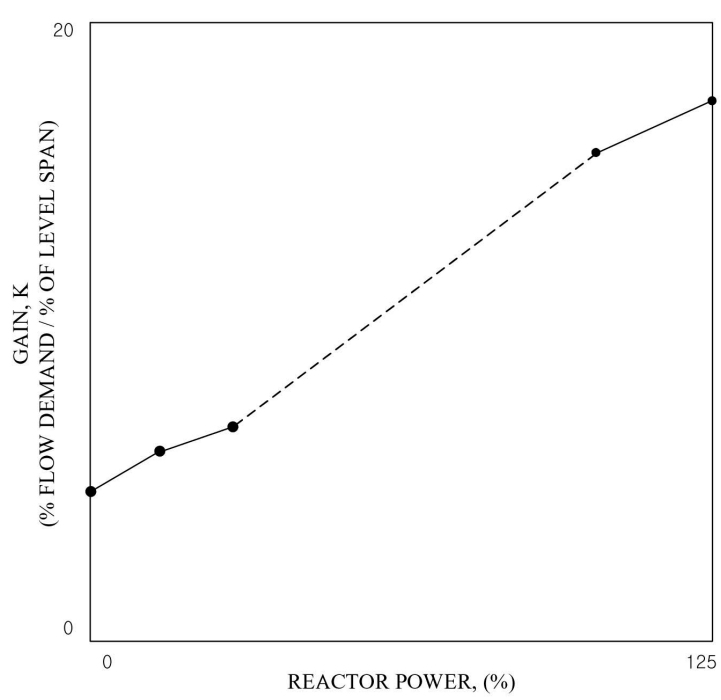

Fig. 4. Gain $K$ for FWCS Master PI Controller

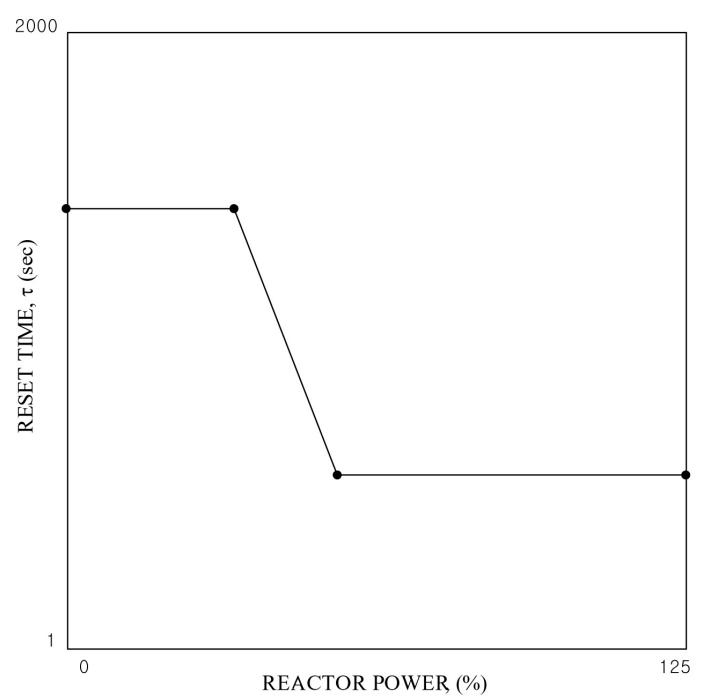

Fig. 5. Reset Time Constant $\tau$ for FWCS Master PI Controller

reset time point) design variables for each optimization for the high and low reactor power region, respectively. 
The objective functions (optimization indexes) evaluated in this work are the degree of the SG level deviation from the target level setpoint and the difference between the two SG (SG \#1 and SG \#2) levels, in accordance with the values of the FWCS parameters. These can be evaluated by computer simulation as follows:

Firstly, at time $t$, an instant SG level deviation $f_{L V D}(t)$ is evaluated as the difference between an instant SG level $L V$ and the target level $L V_{\text {setpoint }}$. The instant SG level deviation is evaluated for both SGs according to the Eq. (3).

$$
\begin{aligned}
& f_{L V D, S G \# 1}(t)=\left|L V_{S G \# 1}(t)-L V_{\text {setpo int }}\right| \\
& f_{L V D, S G \# 2}(t)=\left|L V_{S G \# 2}(t)-L V_{\text {setpo int }}\right|
\end{aligned}
$$

Then, the index for the SG level deviation is evaluated as the average of the instant SG level deviation for $n$ periods as shown in the Eq. (4).

$$
g_{L V D}\left(x_{1}, x_{2}, x_{3}, x_{4}, x_{5}, x_{6}\right)=\frac{\sum_{k=1}^{n}\left\{f_{L V D, S G \# 1}(k \Delta t)+f_{L V D, S G \# 2}(k \Delta t)\right\}}{n} \text {, }
$$

where $\left(x_{1}, x_{2}, x_{3}, x_{4}, x_{5}, x_{6}\right)$ are the values of the piecewise linear gain points and that of reset time point. Also, $\Delta t$ indicates the time interval for the computer simulation, $n \Delta t$ indicates the end of simulation time, and $k \Delta t,(1 \leq k \leq$ $n)$ is the time from the start of the simulation at the $k$ th step. In the above formulas, $L V_{S G H 1}(t)$ and $L V_{S G Z_{2}}(t)$ cannot be calculated analytically but can be evaluated by computer simulation.

Secondly, an instant SG level difference $f_{L V_{-} D I F F}(t)$ between the SG \#1 and SG \#2 is evaluated as in Eq. (5).

$$
f_{L V_{-} D I F F}(t)=\left|L V_{S G \# 1}(t)-L V_{S G \# 2}(t)\right|
$$

The index for the SG level difference between the two SGs is evaluated as the average of the instant SG level difference for $n$ periods like as the Eq. (6).

$$
g_{L V_{-} D I F F}\left(x_{1}, x_{2}, x_{3}, x_{4}, x_{5}, x_{6}\right)=\frac{\sum_{k=1}^{n} f_{L V_{-} D I F F}(k \Delta t)}{n}
$$

In the above explanation, equations (4) and (6) are the objective functions of this optimization and are the relationships between the SG level and the FWCS parameters. These are not analytically calculated because the values of $f_{L V D, S G \# 1}(k \Delta t), f_{L V D, S G \# 2}(k \Delta t), f_{L V-D I F F}(k \Delta t)$ can be evaluated by computer simulation after inputs $\left(\left(x_{1}, x_{2}, x_{3}, x_{4}, x_{5}, x_{6}\right)\right.$; values of FWCS parameters) are determined.
Because there are two objective functions, this work becomes a multiple objective optimization. To solve this problem, the use of desirability functions by which the researcher's own priorities and desires on the response value are expressed for the optimization procedure is considered. One- and two-sided desirability functions are used depending on whether the response is to be maximized or minimized or has an assigned target value [4].

The following desirability functions are used: The first index is the degree of the SG level deviation from the setpoint and the second index is the degree of the SG level difference between the two SGs. For index1, from the viewpoint of the SG level deviation from the set point, the deviation value may be $-44 \% \sim+56 \%$ and the maximum deviation value (absolute value) is chosen as $50 \%$. The value of ' 0 ' is given as the desirability function value for $50 \%$ deviation (the worst case) and the value of ' 1 ' is given for $0 \%$ deviation (the best case). For deviations between $0 \%$ and $50 \%$, values between ' 0 ' and ' 1 ' are assigned. The desirability function has the typical form of $d_{i}=\left(\frac{\hat{y}-y_{0}}{y_{1}-y_{0}}\right)^{n}[4]$. Therefore, the desirability function for the index 1 is given as Fig. 6. For index2, from the viewpoint of the level difference between the two SGs, the difference value may be $-100 \% \sim+100 \%$. A difference of $0 \%$ is the best case and a difference of $100 \%$ is the worst case. The desirability function for the index 2 is given as Fig. 7 so that the degree of the $\mathrm{SG}$ level difference between the two SGs may be forced to go to zero through the optimization. Then, a single composite response $D$ is calculated according to Eq. (7).

$$
D=\sqrt{d_{1} \cdot d_{2}}
$$

Taking into account the two responses, clearly one wishes to choose the condition on the design variable (the values of the piecewise linear gain points and that of the reset time point in this work) that maximizes $D$. A value of $D$ close to 1.0 implies that all responses are in a desirable range simultaneously.

Then, the optimization that maximizes the value of $D$ is performed in the following conditions: As mentioned above, the optimization of the gain and the reset time for high reactor power and low reactor power are performed separately. The design variables $x_{1}, x_{2}, x_{3}, x_{4}, x_{5}, x_{6}$ are the values of the piecewise linear gain points and that of the reset time point of the FWCS main PI controller. The initial value of $\mathrm{x}=\left(x_{1}, x_{2}, x_{3}, x_{4}, x_{5}, x_{6}\right)$ is chosen as the existing values of the gain and reset time for the FWCS of the reference NPP. The first five values are the piecewise linear points of the gain and the last value is that of the reset time. Typically, the region for the variables to be 


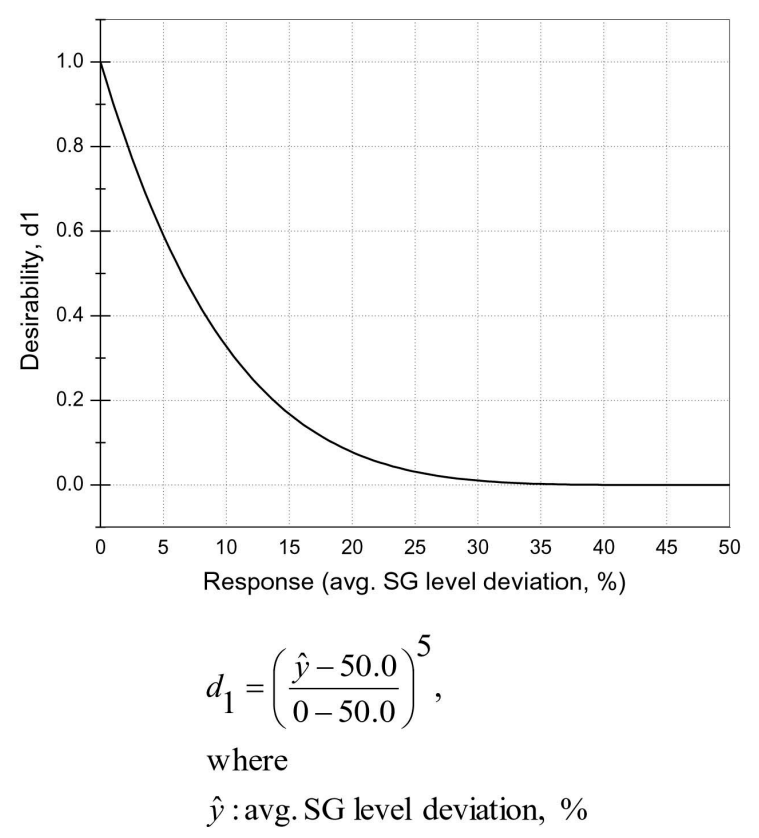

Fig. 6. Desirability Function for Index 1

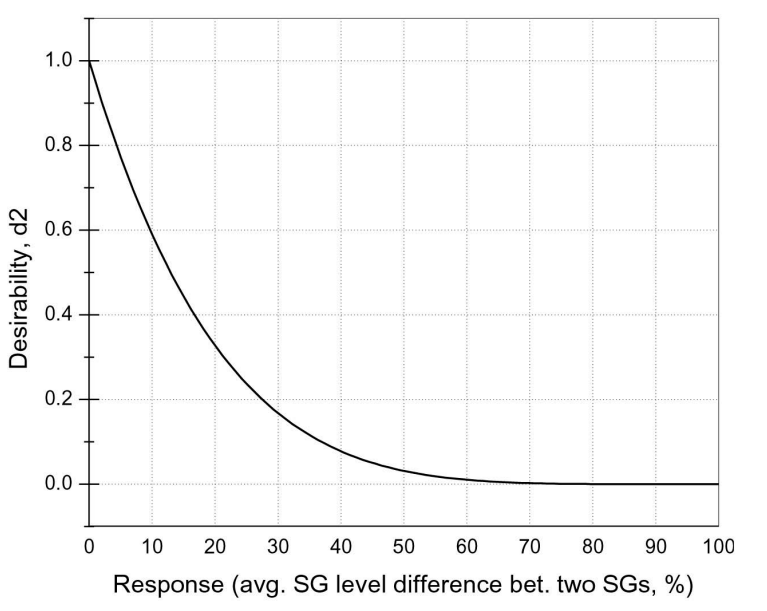

$d_{2}=\left(\frac{\hat{y}-100.0}{0-100.0}\right)^{5}$,

where

$\hat{y}$ :avg. SG level difference bet. two SGs, $\%$

Fig. 7. Desirability Function for Index 2

optimized is mapped into [0, 1]. Also the size for the region of interest is determined as one tenth of the region for the variables. Therefore, the size for the region of interest is set to 0.1 . In the phase one of the RSM procedure, a $2^{6}$ full factorial (resolution VI) design [4] is used to establish the first order model and each established first order model is tested whether these models reflect the real response well or not. The method of steepest ascent is used to determine the search direction. In phase two, a central composite design (CCD) [4] is used to fit the second order model. For computer simulations, the KISPAC code [5] is utilized. The KISPAC code is a best-estimate nuclear power plant simulation tool that analyzes the thermalhydraulic response of the nuclear steam supply system (NSSS) of the OPR1000 type nuclear power plant to a wide variety of plant transients. These include non-LOCA accidents, load maneuvering (steps and ramps), plant trips, and plant heatup/cooldown. The code can be used for overall power plant design, NSSS control system design, and engineering analysis of plant transients.

The following four transients were selected as simulation cases for the optimization. These are the representative transient cases from the view point of the FWCS operation in NPPs. The first three transients correspond to high reactor power and the last one is related to low reactor power:

(a) Power ramp down from $100 \%$ to $20 \%$ during a period of $2000 \mathrm{secs}$

(b) Load rejection to house load (from 100\% to 5\% turbine power)

(c) Loss of a main feedwater pump at $100 \%$ of power

(d) Power oscillation in low range (5\%-18\%-5\% power)

The UCN 5 and 6 were selected as reference NPPs; hence, the SG level setpoint is $44 \%$. The optimization indexes are evaluated by simulating the transients and the optimization results are shown as follows:

Fig. 8 and Fig. 9 show the variation of the $D$-value. For the high reactor power region, all three high power transients ((a), (b), (c)) were simulated and evaluated at each step. The initial $D$-value was evaluated as 0.4439 , which is not a desirable value. As the optimization progressed, the $D$-value moved toward 1.0 and became 0.8829 after 1889 search steps. This implies that the two responses (the degree of the SG level deviation from the setpoint and the degree of the SG level difference between the two SGs) are in a desirable range simultaneously. For the low reactor power region, the low power transients (d) were simulated and evaluated at each step. The initial $D$-value was evaluated as 0.9010 ; this value is almost desirable. Through the optimization process, the $D$-value slightly increased and became 0.9353 in 395 search steps. That is, the effect of the optimization for the low reactor power region is small compared to that of the high reactor power region.

The increase of the $D$-value implies that the two responses of objective functions become more desirable simultaneously; this is shown in Fig. 10 and Fig. 11. The average SG level deviation from the setpoint was minimized from $13.6774 \%$ to $2.3104 \%$ and the average level difference between the two SGs changed from $0.2633 \%$ to $0.1245 \%$ for the high reactor power region. For the low reactor power region, the average SG level deviation has an initial value of $1.9675 \%$, which was minimized to $1.2774 \%$. The average level difference changed from $0.2604 \%$ to $0.2708 \%$. As 


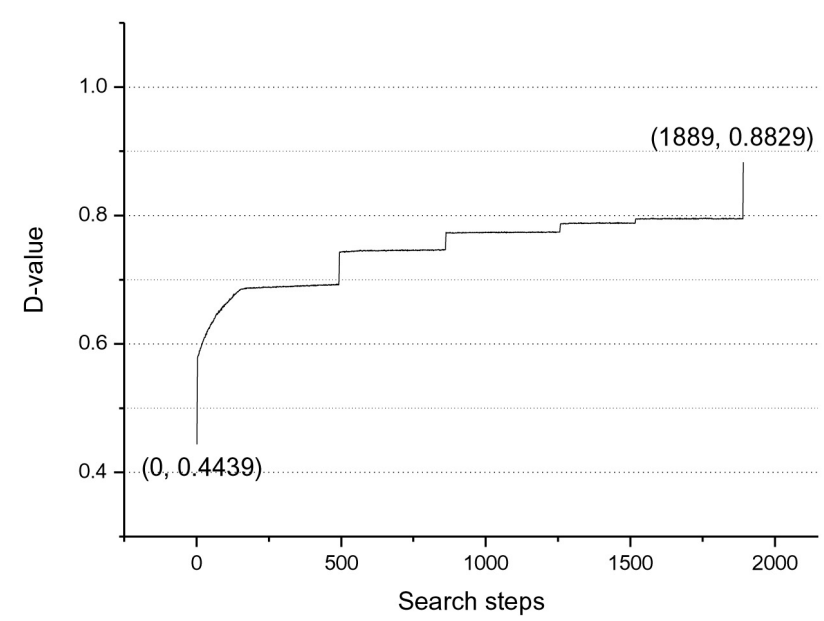

Fig. 8. Variation of D-value for High Reactor Power Region

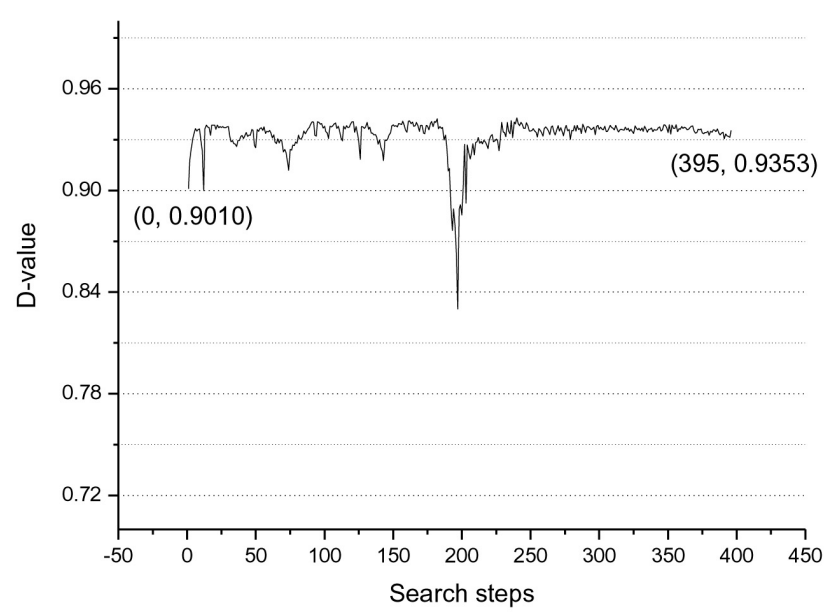

Fig. 9. Variation of D-value for Low Reactor Power Region

mentioned before, for the low reactor power region, the existing gain and reset time were evaluated as almost desirable and the optimization cannot provide large effect for this region.

At the end of the first stage, for both regions, the second order model was generated and evaluated once only and the point maximizing the $D$-value was obtained. Through optimization, the FWCS parameter values were changed to the value indicated in Fig. 12 and Fig. 13. There are a total of twelve variables such as the six variables for the high power region and the six variables for the low power region. The optimization for the high power region and that for the low power region are performed separately, optimizing six variables for each optimization, as mentioned before. Figs. 12 and 13 show the total twelve optimized points together corresponding to the reactor power.

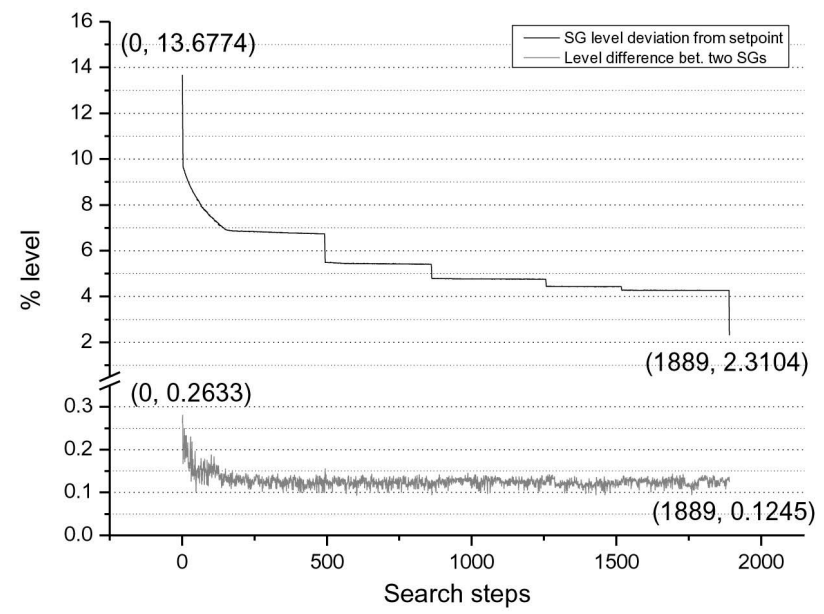

Fig. 10. Variation of Average Level Deviation from Setpoint and Level Difference between Two SGs for High Reactor Power Region

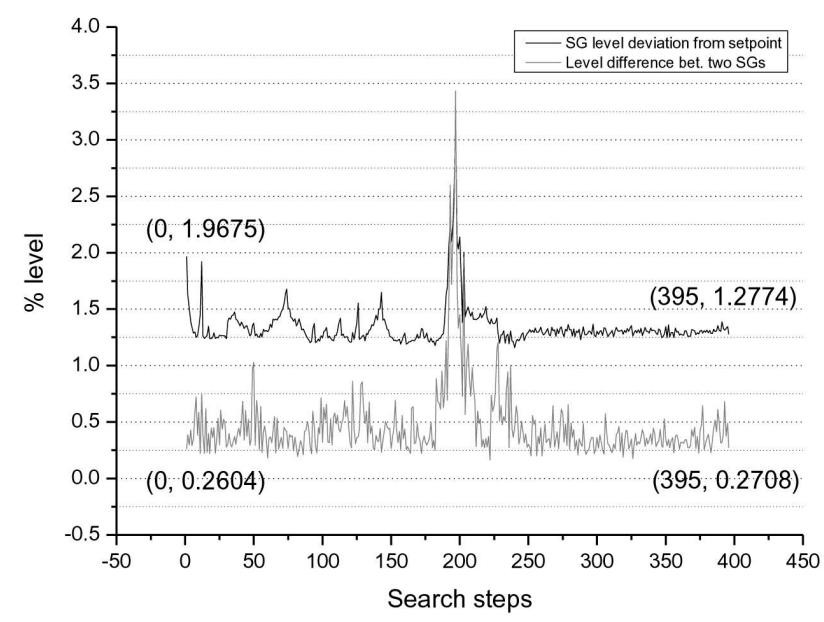

Fig. 11. Variation of Average Level Deviation from Setpoint and Level Difference between Two SGs for Low Reactor Power Region

\subsection{Results of Analysis}

The behaviors of the SG level during the four above mentioned transients are shown in Figs. 14-17. These results show the effect of the optimization. During the power ramp down from $100 \%$ to $20 \%$, one of two feedwater pumps is turned off when the power decreases under $50 \%$ power, and the feedwater flow decreases for an instant. This creates an SG level variation. As shown in Fig. 14, when the existing parameters are used, the SG level varies largely from the level setpoint (44\%) during the power ramp down. However, after optimization of the parameters, the degree of the SG level variation is minimized. Fig. 15 and Fig. 16 show the SG level during load rejection to house load and loss of a main feedwater pump event, respectively. These figures show similar results. In these figures, the 


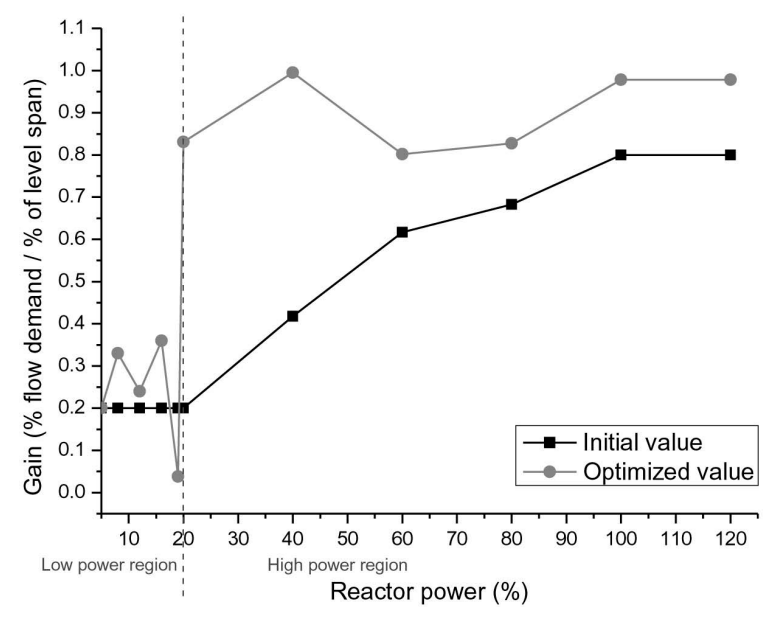

Fig. 12. Optimization Result for Master PI Controller Gain $K$

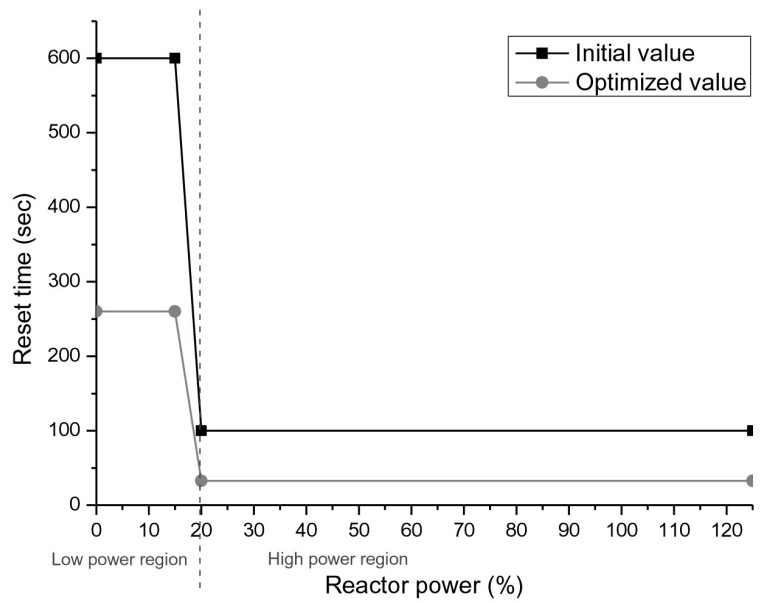

Fig. 13. Optimization Result for Master PI Controller Reset Time $\tau$

sudden decrease of the SG level in the early stage is due to the characteristics of the main steam and feedwater system of the nuclear power plant; the FWCS has an important role in recovering and regulating this distorted SG level. After optimization, the amount of the SG level deviation from the level setpoint is considerably decreased. Fig. 17 does not show the large difference between the existing case and the case in which optimized parameter were used. This is, as expected, because the existing gain and reset time for the low reactor power region are evaluated as almost desirable and the optimization cannot provide a large improvement.

From these results, it has been shown that the control performance of the FWCS is remarkably improved. That is, the parameter optimization provides optimal performance for the FWCS.

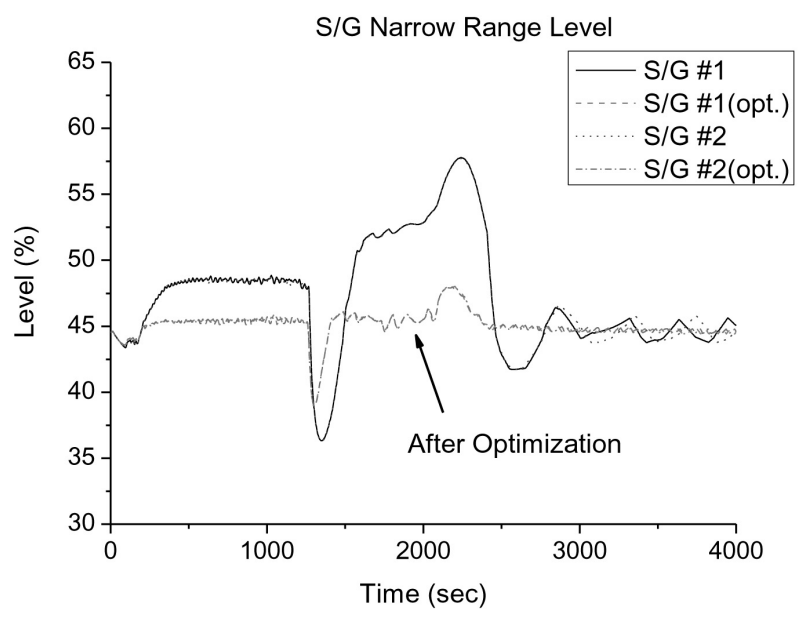

Fig. 14. SG Level during Power Ramp Down from $100 \%$ to $20 \%$

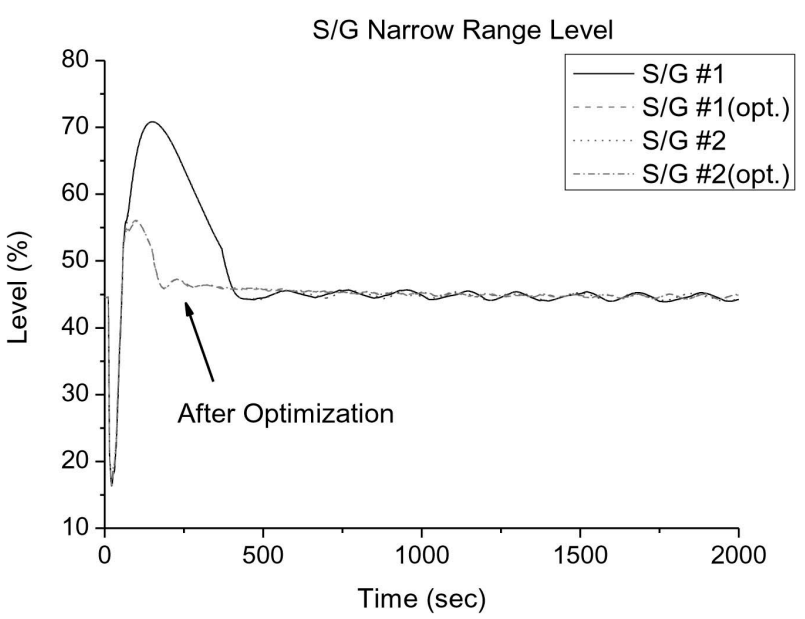

Fig. 15. SG Level during Load Rejection to House Load 5\%

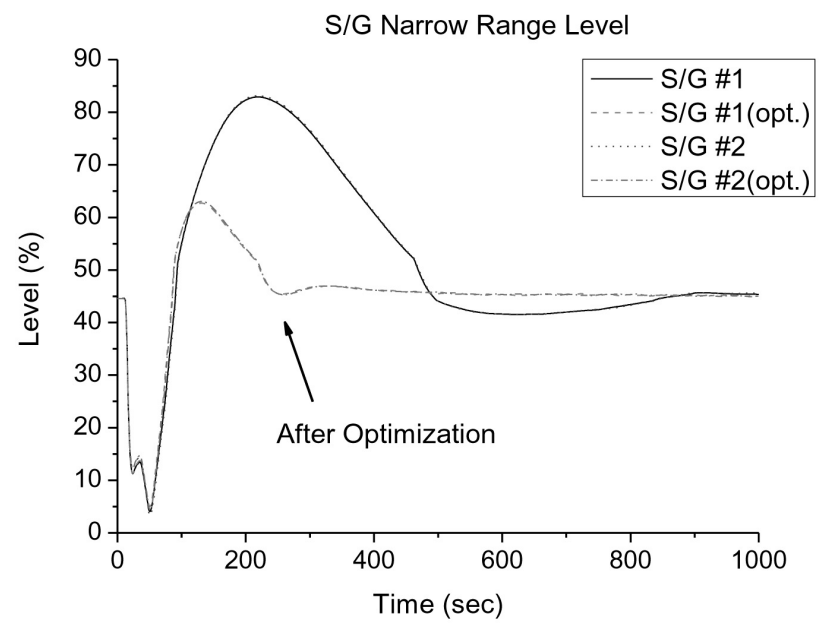

Fig. 16. SG Level during Loss of a Main Feedwater Pump 


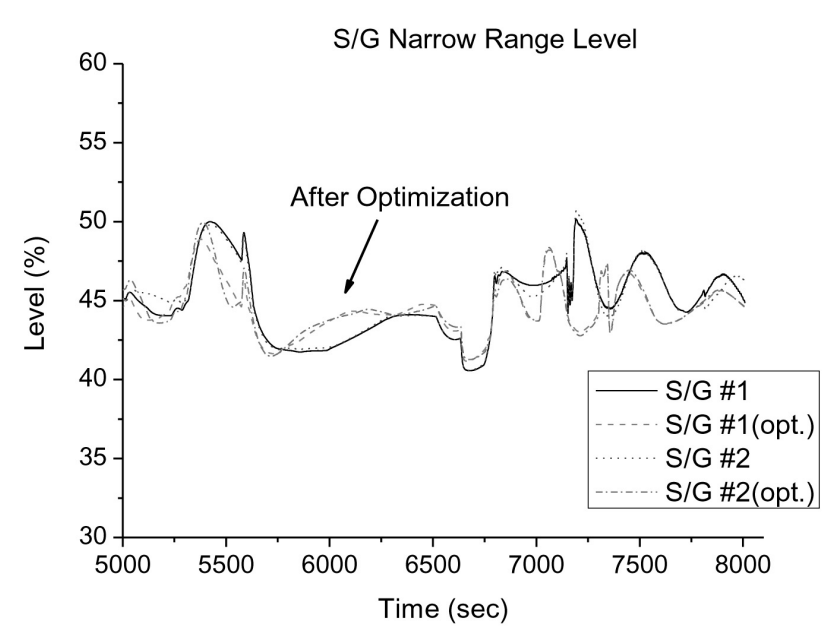

Fig. 17. SG Level during Low Power Oscillation (5\%-18\%-5\%)

\section{SUMMARY AND CONCLUSIONS}

The optimization of the parameters for the FWCS was performed to provide the FWCS with better SG level control performance for UCN 5 and 6. The objective of the optimization was to minimize the SG level deviation from the reference level during transients in NPP operation. The objective functions for this optimization are the relationships between the $\mathrm{SG}$ level deviation from the reference level and the parameters of the FWCS. Since these objective functions are not available in the form of analytic equations, the response for input can only be evaluated by computer simulation using the NPP system simulation code and the simulation optimization methodology was used. In the simulation optimization, the responses of the objective functions and related constraints are evaluated by computer simulation. Among the several simulation optimization methods, the RSM was used for the optimization of the parameters of the FWCS. The parameters to be optimized are the gain and reset time of the FWCS master PI controller. These parameters were optimized for the high and low reactor power region separately. Four representative transients from the view point of the FWCS operation were selected as simulation cases for the optimization. The objective functions were evaluated using a system simulation computer code that has been utilized for the system performance analysis of actual NPPs.

The result shows that the optimized parameters have better SG level control performance. The amount of SG level deviation from the reference level during transients was minimized and consequently the control performance of the FWCS was remarkably improved. This improved SG level control performance results in several affirmative effects. It may help stable operation of the NPP and decrease the possibility of an $\mathrm{SG}$ level related reactor trip. The contribution to maintaining the mechanical integrity of the feedwater valves and pumps is another merit. In addition, the improved SG level control performance may mitigate NPP operator's mental stress during transients. Also, it is concluded that this parameter optimization method can be applied to other NSSS control systems such as the steam bypass control system (SBCS), the reactor regulating system (RRS), the pressurizer level control system (PLCS), the pressurizer pressure control system (PPCS) and so on, and can be utilized in the actual design of NPPs.

\section{REFERENCES}

[1 ] Y. Carson and A. Maria, "Simulation Optimization: Methods and Applications," Proceedings of the 1997 Winter Simulation Conference, p.118-126 (1997).

[2] F. Azadivar, "Simulation Optimization Methodologies," Proceedings of the 1999 Winter Simulation Conference, p.93-100 (1999).

[ 3 ] H. Gonda Neddermeijer, G. J. van Oortmarssen, N. Piersma, and R. Dekker, "A Framework for Response Surface Methodology for Simulation Optimization," Proceedings of the 2000 Winter Simulation Conference, 129-136 (2000).

[4] R. H. Myers and D. C. Montgomery, Response Surface Methodology: Process and Product Optimization Using Designed Experiments, John Wiley \& Sons, New York (1995).

[ 5 ] U. S. Kim, S. W. Sohn, and I. H. Song, "PI Controllers in Performance Analysis Computer Code for Nuclear Power Plants," Transactions of the Korean Nuclear Society Autumn Meeting, PyeongChang, Korea, October 25-26 (2007). 